\title{
Contributions of prescribed and non-prescribed opioids to opioid related deaths: population based cohort study in Ontario, Canada
}

\author{
Tara Gomes, ${ }^{1}$ Wayne Khuu, ${ }^{2}$ Diana Martins, ${ }^{1}$ Mina Tadrous, ${ }^{1}$ Muhammad M Mamdani, ${ }^{3}$ \\ J Michael Paterson, ${ }^{2}$ David N Juurlink ${ }^{4}$
}

${ }^{1}$ Li Ka Shing Knowledge Institute of St. Michael's

Hospital, Toronto, ON, Canada

${ }^{2}$ Institute for Clinical Evaluative Sciences, Toronto, ON, Canada

${ }^{3} \mathrm{Li}$ Ka Shing Centre for

Healthcare Analytics Research

and Training at St. Michael's

Hospital, Toronto, ON, Canada

${ }^{4}$ The Sunnybrook Research Institute, Toronto, ON, Canada

Correspondence to:

T Gomes GomesT@smh.ca

(or @Tara_Gomes on Twitter)

Additional material is published online only. To view please visit the journal online.

Cite this as: $B M J$ 2018;362:k3207 http://dx.doi.org/10.1136/bmj.k3207

Accepted: 9 July 2018

\section{ABSTRACT}

OBJECTIVE

To describe the contributions of prescribed and nonprescribed opioids to opioid related deaths.

DESIGN

Population based cohort study.

SETTING

Ontario, Canada, from 1 January 2013 to 31 December 2016.

\section{PARTICIPANTS}

All Ontarians who died of an opioid related cause.

\section{EXPOSURE}

Active opioid prescriptions, defined as those with a duration overlapping the date of death, and recent opioid prescriptions, defined as those dispensed in the 30 and 180 days preceding death. Postmortem toxicology results from the Drug and Drug/Alcohol Related Death database were used to characterise deaths on the basis of presence of prescribed and non-prescribed (that is, diverted or illicit) opioids, overall and stratified by year and age.

\begin{abstract}
RESULTS
2833 opioid related deaths occurred. An active opioid prescription on the date of death was relatively common but declined slightly throughout the study period $(38.2 \%(241 / 631)$ in 2013 and $32.5 \%$ $(278 / 855)$ in 2016; $P$ for trend=0.03). Older people and women were relatively more likely to have an active opioid prescription at time of death. In 2016 , $46 \%(169 / 364)$ of people aged 45-64 had an active opioid prescription compared with only $12 \%(8 / 69)$ among those aged 24 or younger ( $P$ for trend 0.001 ). Similarly, $46 \%(124 / 272)$ of women had an active opioid prescription at time of death compared with $26.4 \%(154 / 583)$ of men ( $\mathrm{P}<0.001)$. Among people with active opioid prescriptions at time of death,
\end{abstract}

\section{WHAT IS ALREADY KNOWN ON THIS TOPIC}

Opioid related deaths continue to climb in North America

This growing problem has been associated with the widespread prescribing of opioids over the past 20 years

Use of non-prescribed (diverted or illicit) opioids is thought to be the major contributor to opioid related deaths

\section{WHAT THIS STUDY ADDS}

Prescribed, diverted, and illicit opioids all play an important role in opioid related deaths

One third of opioid related deaths were among people actively treated with a prescription opioid

A concerning trend of increased involvement of non-prescribed fentanyl in 2016 aligns with the recent introduction of illicit fentanyl to the market
$37.8 \%(375 / 993)$ also had evidence of a nonprescribed opioid on postmortem toxicology. By 2016, the non-prescribed opioid most commonly identified after death was fentanyl (41\%; 47 of 115 cases). Among people without an active opioid prescription at time of death, fentanyl was detected in $20 \%$ (78/390) of deaths in 2013 , increasing to $47.5 \%(274 / 577)$ by 2016 ( $P<0.001)$.

\section{CONCLUSIONS}

Prescribed, diverted, and illicit opioids all play an important role in opioid related deaths. Although more than half of all opioid related deaths still involved prescription drugs (either dispensed or diverted) in 2016, the increased rate of deaths involving fentanyl between 2015 and 2016 is concerning and suggests the need for a multifactorial approach to this problem that considers both the prescribed and illicit opioid environments.

\section{Introduction}

The North American opioid overdose crisis has been attributed, in part, to the widespread use of prescription opioids to treat chronic non-cancer pain over the past two decades. ${ }^{1}$ In particular, long term and high dose opioid prescribing has been associated with serious adverse events, including risks of developing an opioid use disorder, overdose, and death. ${ }^{1-3}$ Furthermore, combined use of opioids with other depressants of the central nervous system, most commonly benzodiazepines, has been highlighted as an important risk factor for death due to overdose. ${ }^{4}$ Despite recent evidence of decreasing rates of opioid prescribing across Canada, $^{5}$ the prevalence of exposure to opioids remains high with an opioid being prescribed to one in eight Ontarians to manage pain in $2016 .^{6}$ Similarly, more than 42000 opioid related deaths (13.1 per 100000 population) are estimated to have occurred in the United States and nearly 3000 in Canada (8.1 per 100000 population) in that year. $^{78}$ The burden of this early loss of life is clear. In Ontario, the most populous province in Canada, 1 in 133 deaths in 2015 were opioid related, leading to nearly 30000 years of life lost. ${ }^{9}$ Since this time, the policy response has primarily focused on improving the care of people with opioid use disorders, reducing diversion of prescribed opioids, and promoting safe, appropriate opioid prescribing, as evidenced by new clinical practice guidelines for opioids in chronic noncancer pain published for both the US and Canada in 2016 and 2017..$^{1011}$

Shifts in prescribed opioid formulations, ${ }^{5}$ restrictions on accessibility of prescription drugs, ${ }^{12}$ and the growing presence of clandestinely manufactured 
opioids have caused the illicit opioid market to become considerably more toxic since 2012. ${ }^{13} 14$ In particular, the emergence of clandestinely manufactured fentanyl (and its analogues) has been associated with a sharp increase in opioid related fatalities in parts of North America, as these potent drugs are contaminating the illicit opioid market. ${ }^{15-18}$ For example, in British Columbia, Canada, 1156 deaths due to drug overdose involving illicit fentanyl occurred in 2017, representing a $73 \%$ increase over the same period the previous year. ${ }^{18}$ In Ontario, a $62 \%$ increase in the rate of fentanyl related deaths was observed between 2015 and 2016. ${ }^{19}$ Consequently, researchers and policy makers have called for timely data on trends in the relative contributions of prescribed and nonprescribed (that is, diverted and illicit) opioids to opioid related deaths. ${ }^{2021}$ This information can be a crucial component of evidence informed policy responses that comprehensively address the roles that prescribers, pharmacists, harm reduction workers, and law enforcement can play in tackling the ongoing opioid crisis. $^{22} 23$

An understanding of this problem requires linked data from both prescription claims and postmortem toxicological investigations-data that are rarely available at a population level. Here, we used two such databases to study the relative contributions of prescribed and non-prescribed opioids to opioid related deaths in Ontario, the most populous province in Canada. Specifically, our primary objective was to estimate the proportion of people dying of an opioid related cause who had an active opioid prescription at time of death and to evaluate how this has changed over time.

\section{Methods}

\section{Setting}

We conducted a population based cohort study of all people who died of an opioid related cause in Ontario between 1 January 2013 (when sufficient comprehensive data on all opioid prescriptions were available in the province) and 31 December 2016. In Ontario, a medical coroner investigates all deaths that are sudden or unexpected to determine the cause of death. Deaths to which an opioid contributed, either alone or in combination with another drug or alcohol, are defined as opioid related and included in this analysis.

\section{Data sources}

We used the Drug and Drug/Alcohol Related Death (DDARD) database to identify all deaths determined to be opioid related in Ontario over the study period. This database is created through chart abstraction of coronial investigations and includes details on manner of death (accidental, suicide, undetermined) and drug concentrations from postmortem toxicology (see supplementary materials for opioids captured in postmortem toxicology). ${ }^{24}$ We used the Ontario Health Insurance Plan (OHIP) Registered Persons Database, which contains information on every Ontarian ever issued a health card, to identify demographic information and the Ontario Narcotics Monitoring System (NMS) database to identify all prescriptions for opioids and benzodiazepines dispensed from community pharmacies. This database includes all prescriptions dispensed, regardless of payer. We also used the Ontario Drug Benefit (ODB) claims database to identify publicly funded prescription drugs dispensed to eligible residents of Ontario over the study period, and the OHIP Claims History Database to identify services rendered by physicians. We identified all diagnoses and procedures occurring in emergency departments and hospital admissions by using the Canadian Institute for Health Information's National Ambulatory Care Reporting System and Discharge Abstract Database, respectively. All datasets were linked using unique, encoded identifiers, and were analysed at the Institute for Clinical Evaluative Sciences (ICES; www.ices.on.ca).

\section{Identification of patients}

We identified all people who died of an opioid related cause over our study period and excluded those with invalid patient identifiers who could not be linked to the ICES data repository. In all analyses, we defined the index date as the date of death.

\section{Opioid exposure definition}

In our primary analysis, we defined active opioid prescriptions as those with a day's supply overlapping the index date. In a secondary analysis, we defined recent opioid prescriptions as those dispensed in the 30 days, 180 days, one year, two years, and three years before the index date. To investigate the contribution of benzodiazepines to opioid related deaths, we defined active benzodiazepine prescriptions as those with a day's supply overlapping the index date and measured the prevalence of concomitant active prescriptions for both opioids and benzodiazepines. In a sensitivity analysis to explore the role of fentanyl in any temporal changes in prevalence of active opioid prescriptions, we replicated our primary analysis, excluding all deaths for which fentanyl was present on postmortem toxicology. Finally, in a post hoc secondary analysis to test the generalisability of these findings to nonfatal overdoses, we replicated our analysis among people visiting the emergency department with an opioid related toxicity by using ICD-10 (International Classification of Diseases, 10th revision) diagnosis codes T40.0, T40.1, T40.2, T40.3, T40.4, and T40.6.

\section{Patient characteristics}

We used linked databases to identify sociodemographic characteristics, including age, sex, urban/rural location of residence, and fifth of neighbourhood income. Furthermore, we determined the prevalence of previous diagnoses of mental health problems and addictions, including alcohol use disorder (previous three years), hospital visits (emergency department visit or inpatient hospital admission) for an opioid overdose (previous year), and emergency department visits for 
other mental health diagnoses (anxiety disorders, deliberate self harm, mood disorders, schizophrenia, and substance related disorders; previous three years). See the supplementary materials for the ICD-10 codes we used to define each of these comorbidities. Finally, we used postmortem toxicology to characterise involvement of other drugs in opioid related deaths, including cocaine, benzodiazepines, antipsychotics, antihistamines, stimulants, tetrahydrocannabinol, tricyclic antidepressants, and other antidepressants.

\section{Statistical analysis}

We summarised patients' characteristics over the study period by using descriptive statistics, and we stratified the analysis according to whether the patient had an active opioid prescription at time of death. We used $\chi^{2}$ and one way analysis of variance test statistics to compare categorical and continuous characteristics, respectively, between cohorts. In our primary analysis, we reported the prevalence of active and recent opioid prescriptions and active concomitant benzodiazepine prescriptions before opioid related deaths in Ontario. In a secondary analysis, we measured the prevalence of active opioid prescriptions, stratified by age and sex. Finally, we studied the relative contribution of prescribed and non-prescribed opioids to each death in each year of the study period by using postmortem toxicology results. We used two tailed, exact CochranArmitage trend tests to determine whether significant trends existed in the prevalence of prescribed and non-prescribed opioids over the study period and the Kruskal-Wallis test to compare medians over time. We adjusted all postmortem drug concentrations for the potential prevalence of opioid metabolites wherever possible (see supplementary materials for details of these adjustments). In particular, we determined heroin involvement on the basis of the presence of 6-monoacetylmorphine in postmortem toxicology. We used SAS software (version 9.4) for all analyses and a type 1 error rate of 0.05 as the threshold for statistical significance.

\section{Patient involvement}

No patients were involved in setting the research question or the outcome measures, nor were they involved in developing plans for design or implementation of the study. No patients were asked to advise on interpretation or writing up of results. There are no plans to disseminate the results of the research to study participants. However, study results will be disseminated to patients and citizens involved in the Ontario Drug Policy Research Network Citizens' Panel and the Ontario Opioid Drug Observatory.

\section{Results}

We identified 2910 opioid related deaths in Ontario over the study period, of which 2833 (97.4\%) had valid identifiers permitting their analysis. On average, decedents were 43 (SD 13.5) years of age, 67.5\% $(\mathrm{n}=1911)$ were male, and 34.1\% $(\mathrm{n}=966)$ resided in the neighbourhood with the lowest fifth of income (table 1). Previous diagnoses of mental health disorders and addictions were relatively common, with $8.6 \%(n=244)$ having been admitted to hospital for an opioid overdose in the year before death, 25.8\% $(n=730)$ having a diagnosis of alcohol use disorder, and $41.9 \%(n=1188)$ having a visit to an emergency department for a mental health disorder in the previous three years. Overall, patients' demographic characteristics and involvement of other drugs in death differed considerably according to whether the patient had an active opioid prescription at time of death. In particular, people with no active opioid prescriptions tended to be younger, were more likely to be men, were slightly more likely to reside in higher income neighbourhoods, and were more likely to have cocaine or stimulants present in postmortem toxicology at time of death (table 1). In contrast, we observed little difference in the patterns of use of health services, diagnoses of alcohol use disorder, and mental health diagnoses between patient groups.

In 2013, 38.2\% (241/631) of people who died from an opioid related cause had an active opioid prescription on their date of death. Although the absolute number of deaths involving an active prescription increased by $15.4 \%$ between 2013 and 2016 (from 241 to 278 deaths), the proportion of all deaths with an active opioid prescription declined significantly to $32.5 \%(278 / 855)$ in 2016 ( $\mathrm{P}=0.03$; table 2). When considering recent opioid prescribing (previous 30 days), we found a similar trend over time, but these reductions did not achieve statistical significance. In contrast, we observed no apparent trend in the prevalence of more remote opioid prescribing (from six months to three years before the index date). By 2016, 59.4\% ( $n=508)$ had received an opioid in the preceding 180 days, rising to $76.4 \%$ $(n=653)$ when we considered a three year lookback period. Although prevalence declined over our study period, active benzodiazepine prescriptions remained common in 2016, with $27.6 \% \quad(n=236)$ of people who died of an opioid related cause having an active benzodiazepine prescription at time of their death and $16.1 \%(n=138)$ having active prescriptions for both benzodiazepines and opioids. In our sensitivity analysis, we found that, after exclusion of deaths involving fentanyl, a significant change in the prevalence of active opioid prescriptions at time of opioid related death (36.8\% in 2013 v 39.8\% in 2016; $\mathrm{P}=0.18$ ) was no longer apparent. This suggests that the changes in trend observed in our primary analysis were being driven by deaths involving fentanyl.

In our analysis stratified by age and sex, people aged 45 years and older and women were more likely to have an active opioid prescription at their time of death (table 3). In particular, 46\% (169/364) of patients aged 45-64 had an active opioid prescription in 2016 compared with only 12\% (8/69) among those aged 24 or younger $(\mathrm{P}<0.001$; table 3$)$. Similarly, $46 \%$ $(124 / 272)$ of women had an active opioid prescription at time of death compared with $26.4 \%$ (154/583) of men $(\mathrm{P}<0.001$; table 3$)$. 


\begin{tabular}{|c|c|c|c|c|}
\hline Characteristics & $\begin{array}{l}\text { Opioid related death } \\
(n=2833)\end{array}$ & $\begin{array}{l}\text { No active opioid prescription } \\
(n=1836)\end{array}$ & $\begin{array}{l}\text { Active opioid prescription } \\
(\mathrm{n}=997)\end{array}$ & Pvalue* \\
\hline Mean (SD) age, years & $43.0(13.5)$ & $39.8(13.2)$ & $49.0(11.8)$ & $<0.001$ \\
\hline \multicolumn{5}{|l|}{ Age group, years: } \\
\hline $0-24$ & $240(8.5)$ & $220(12.0)$ & $20(2.0)$ & \multirow{5}{*}{$<0.001$} \\
\hline $25-34$ & $647(22.8)$ & $537(29.2)$ & $110(11.0)$ & \\
\hline $35-44$ & $596(21.0)$ & $402(21.9)$ & $194(19.5)$ & \\
\hline $45-64$ & $1222(43.1)$ & $622(33.9)$ & $600(60.2)$ & \\
\hline$\geq 65$ & $128(4.5)$ & $55(3.0)$ & $73(7.3)$ & \\
\hline \multicolumn{5}{|l|}{ Sex: } \\
\hline Female & $922(32.5)$ & $493(26.9)$ & $429(43.0)$ & \multirow{2}{*}{$<0.001$} \\
\hline Male & $1911(67.5)$ & $1343(73.1)$ & $568(57.0)$ & \\
\hline \multicolumn{5}{|l|}{ Fifth of neighbourhood income: } \\
\hline 1 (lowest) & $966(34.1)$ & $574(31.3)$ & $392(39.3)$ & \multirow{6}{*}{$<0.001$} \\
\hline 2 & $591(20.9)$ & 365 (19.9) & $226(22.7)$ & \\
\hline 3 & $441(15.6)$ & $291(15.8)$ & $150(15.0)$ & \\
\hline 4 & $384(13.6)$ & $279(15.2)$ & $105(10.5)$ & \\
\hline 5 (highest) & $359(12.7)$ & $245(13.3)$ & $114(11.4)$ & \\
\hline Missing & $92(3.2)$ & $82(4.5)$ & $10(1.0)$ & \\
\hline \multicolumn{5}{|l|}{ Location of residence: } \\
\hline Rural & $293(10.3)$ & $179(9.7)$ & $114(11.4)$ & \multirow{3}{*}{$<0.001$} \\
\hline Urban & $2484(87.7)$ & $1602(87.3)$ & $882(88.5)$ & \\
\hline Missing & $56(2.0)$ & $55(3.0)$ & $1(0.1)$ & \\
\hline Hospital visit for opioid overdose (previous year) & $244(8.6)$ & $156(8.5)$ & $88(8.8)$ & 0.77 \\
\hline Alcohol use disorder (previous 3 years) & $730(25.8)$ & $477(26.0)$ & $253(25.4)$ & 0.73 \\
\hline Mental health ED visit (previous 3 years): & $1188(41.9)$ & $767(41.8)$ & $421(42.2)$ & 0.82 \\
\hline Anxiety disorders & $339(12.0)$ & $224(12.2)$ & $115(11.5)$ & 0.60 \\
\hline Deliberate self harm & $419(14.8)$ & $269(14.7)$ & $150(15.0)$ & 0.78 \\
\hline Mood disorders & $294(10.4)$ & $185(10.1)$ & $109(10.9)$ & 0.48 \\
\hline Schizophrenia & $122(4.3)$ & $92(5.0)$ & $30(3.0)$ & 0.01 \\
\hline Substance use disorders & $691(24.4)$ & $455(24.8)$ & $236(23.7)$ & 0.51 \\
\hline Other mental health disorders & $82(2.9)$ & $48(2.6)$ & $34(3.4)$ & 0.23 \\
\hline \multicolumn{5}{|l|}{ Presence of other drugs at death on toxicology report: } \\
\hline Cocaine & $925(32.7)$ & $716(39.0)$ & $209(21.0)$ & $<0.001$ \\
\hline Benzodiazepines & $1342(47.4)$ & $748(40.7)$ & $594(59.6)$ & $<0.001$ \\
\hline Antipsychotics & $405(14.3)$ & $211(11.5)$ & $194(19.5)$ & $<0.001$ \\
\hline Antihistamines & $469(16.6)$ & $237(12.9)$ & $232(23.3)$ & $<0.001$ \\
\hline Stimulants & $468(16.5)$ & $367(20.0)$ & $101(10.1)$ & $<0.001$ \\
\hline Tetrahydrocannabinol & $78(2.8)$ & $56(3.1)$ & $22(2.2)$ & 0.19 \\
\hline Tricyclic antidepressants & $250(8.8)$ & $95(5.2)$ & $155(15.5)$ & $<0.001$ \\
\hline Other antidepressants & $1021(36.0)$ & $563(30.7)$ & $458(45.9)$ & $<0.001$ \\
\hline
\end{tabular}

$\mathrm{ED}=$ emergency department.

*P values from $x^{2}$ tests of comparison. P<0.05 indicates statistically significant difference between groups measured.

\begin{tabular}{|c|c|c|c|c|c|}
\hline Measures & $2013(n=631)$ & $2014(n=635)$ & $2015(n=712)$ & $2016(n=855)$ & $P$ value (trend test) \\
\hline Active opioid prescription on date of death & $241(38.2)$ & $224(35.3)$ & $254(35.7)$ & $278(32.5)$ & 0.03 \\
\hline \multicolumn{6}{|l|}{ Opioid prescription within previous: } \\
\hline 30 days & $301(47.7)$ & $283(44.6)$ & $338(47.5)$ & $367(42.9)$ & 0.15 \\
\hline 180 days & $390(61.8)$ & $368(58.0)$ & $432(60.7)$ & $508(59.4)$ & 0.61 \\
\hline 1 year & $-^{\star}$ & $414(65.2)$ & $473(66.4)$ & $555(64.9)$ & 0.87 \\
\hline 2 years & $-^{\star}$ & $-^{\star}$ & $520(73.0)$ & $616(72.0)$ & $0.66 \dagger$ \\
\hline 3 years & $--^{*}$ & $-^{*}$ & $-{ }^{\star}$ & $653(76.4)$ & - \\
\hline Active benzodiazepine prescription on date of death & $207(32.8)$ & $207(32.6)$ & $237(33.3)$ & $236(27.6)$ & 0.04 \\
\hline Active opioid and benzodiazepine prescription on date of death & $127(20.1)$ & $118(18.6)$ & $147(20.6)$ & $138(16.1)$ & 0.10 \\
\hline Sensitivity analysis: active opioid prescription among non-fentanyl & $182 / 494(37)$ & $161 / 464(35)$ & $196 / 500(39)$ & $200 / 503(40)$ & 0.18 \\
\hline
\end{tabular}
opioid related deaths

*Data omitted owing to incomplete data capture in lookback period.

tP value derived from $x^{2}$ test; all other $P$ values reflect two tailed, exact Cochran-Armitage trend tests.

Among the 278 patients who had an active opioid prescription at time of death in 2016, approximately two thirds $(64 \% ; n=179)$ had their opioid prescription reimbursed by the public drug programme, and the median number of days between the date of the last opioid prescription dispensed and the date of the opioid related death was 4 (interquartile range 1-10) days (supplementary materials). Among people with only prescribed opioids detected on postmortem toxicology, the prevalence of specific opioid drugs 


\begin{tabular}{|c|c|c|c|c|c|c|c|c|c|}
\hline \multirow[b]{2}{*}{ Year } & \multicolumn{6}{|c|}{ Age group (years) } & \multicolumn{3}{|l|}{ Sex } \\
\hline & $0-24$ & $25-34$ & $35-44$ & $45-64$ & $\geq 65$ & P value* & Female & Male & P value* \\
\hline 2013 & $\leq 5 / 46$ & $29-33 / 138(21-24)$ & 48/141(34) & $140 / 278(50)$ & $19 / 28(68)$ & $<0.001$ & $101 / 209(48)$ & $140 / 422(33)$ & $<0.001$ \\
\hline 2014 & $\leq 5 / 57$ & $25-29 / 150(17-19)$ & $50 / 142(35)$ & $133 / 267(50)$ & $11 / 19(58)$ & $<0.001$ & $85 / 201(42)$ & $139 / 434(32)$ & 0.01 \\
\hline 2015 & $6 / 68(9)$ & 24/156 (15) & $52 / 140(37)$ & $158 / 313(50)$ & $14 / 35(40)$ & $<0.001$ & $119 / 240(50)$ & $135 / 472(29)$ & $<0.001$ \\
\hline 2016 & $8 / 69(12)$ & $28 / 203(14)$ & $44 / 173(25)$ & $169 / 364(46)$ & $29 / 46(63)$ & $<0.001$ & $124 / 272(46)$ & $154 / 583(26)$ & $<0.001$ \\
\hline
\end{tabular}

\begin{tabular}{|c|c|c|c|c|c|}
\hline Patients with active opioid prescriptiont & $\begin{array}{l}2013 \\
(n=239)\end{array}$ & $\begin{array}{l}2014 \\
(n=223)\end{array}$ & $\begin{array}{l}2015 \\
(n=254)\end{array}$ & $\begin{array}{l}2016 \\
(n=277)\end{array}$ & P value* \\
\hline Only prescribed opioids present on toxicology & $154(64)$ & $142(64)$ & $160(63)$ & $162(58)$ & 0.17 \\
\hline \multicolumn{6}{|c|}{ Type of prescribed opioid that overlapped with death date: } \\
\hline Oxycodone & $56(36)$ & $45(32)$ & $58(36)$ & $53(33)$ & 0.71 \\
\hline Hydromorphoneł & $33(21)$ & $32(23)$ & $48(30)$ & $45(28)$ & 0.09 \\
\hline Fentanyl & $36(23)$ & $32(23)$ & $30(19)$ & $24(15)$ & 0.04 \\
\hline Methadone & $15(10)$ & $10(7)$ & $16(10)$ & $28(17)$ & 0.02 \\
\hline Morphineł & $25(16)$ & $30(21)$ & $19(12)$ & $13(8)$ & 0.01 \\
\hline Codeine & $16(10)$ & $16(11)$ & $10(6)$ & $21(13)$ & 0.81 \\
\hline Other§ & $\leq 5$ & $\leq 5$ & $\leq 5$ & $\leq 5$ & 0.38 \\
\hline Presence of non-prescribed opioids on toxicology & $85(36)$ & $81(36)$ & $94(37)$ & $115(42)$ & 0.17 \\
\hline \multicolumn{6}{|l|}{ Type of opioid on toxicology with no active prescription: } \\
\hline Oxycodone & $11(13)$ & $12(15)$ & $20(21)$ & $13(11)$ & 0.95 \\
\hline Hydromorphoneł & $8(9)$ & $17(21)$ & $18(19)$ & $20(17)$ & 0.25 \\
\hline Fentanyl & $15(18)$ & $16(20)$ & $22(23)$ & $47(41)$ & $<0.001$ \\
\hline Methadone & $26(31)$ & $16(20)$ & $11(12)$ & $12(10)$ & $<0.001$ \\
\hline Morphine $\ddagger$ & $15(18)$ & $9(11)$ & $19(20)$ & $22(19)$ & 0.44 \\
\hline Codeine & $23(27)$ & $24(30)$ & $29(31)$ & $25(22)$ & 0.41 \\
\hline Heroin & $\leq 5$ & $9(11)$ & $8(9)$ & $\leq 5$ & 0.49 \\
\hline Other§ & $\leq 5$ & $\leq 5$ & $\leq 5$ & $\leq 5$ & 1.0 \\
\hline No evidence of active opioid on toxicology & $42(18)$ & $50(22)$ & $41(16)$ & $70(25)$ & 0.12 \\
\hline Patients without active opioid prescription & $(n=390)$ & $(n=411)$ & $(n=458)$ & $(n=577)$ & \\
\hline \multicolumn{6}{|l|}{ Type of opioid on toxicology: } \\
\hline Oxycodone & $86(22)$ & $78(19)$ & $74(16)$ & $96(17)$ & 0.02 \\
\hline Hydromorphonef & $76(19)$ & 79 (19) & $107(23)$ & $104(18)$ & 0.82 \\
\hline Fentanyl & $78(20)$ & $108(26)$ & $154(34)$ & $274(47)$ & $<0.001$ \\
\hline Methadone & $106(27)$ & $89(22)$ & $93(20)$ & $102(18)$ & $<0.001$ \\
\hline Morphine $\neq$ & 76 (19) & 77 (19) & 89 (19) & $97(17)$ & 0.32 \\
\hline Codeine & $83(21)$ & $91(22)$ & $96(21)$ & $67(12)$ & $<0.001$ \\
\hline Heroin & $49(13)$ & $87(21)$ & $76(17)$ & $70(12)$ & 0.24 \\
\hline Other§ & $10(3)$ & $6(1)$ & $7(2)$ & $\leq 5$ & 0.002 \\
\hline \multicolumn{6}{|c|}{$\begin{array}{l}\text { Drugs listed in each group are not mutually exclusive; someone could have multiple non-prescribed drugs present at toxicology, and all are cap } \\
\text { In cases where number of patients is }<6 \text {, this number has been suppressed to ensure confidentiality. In cases where only one record is suppress } \\
\text { range to avoid residual disclosure. } \\
\text { *P values reflect two tailed, exact Cochran-Armitage trend tests. P<0.05 indicates statistically significant difference between groups measured. } \\
\text { †4 patients are excluded from table owing to unknown opioid involvement at time of death. } \\
\text { fOpioids that may be metabolites of other opioids (see note in Methods). } \\
\text { §Includes anileridine, buprenorphine, hydrocodone, meperidine, pentazocine, propoxyphene, and tramadol. }\end{array}$} \\
\hline
\end{tabular}

changed between 2013 and 2016, with fentanyl (from $23 \%$ to $15 \%$; $\mathrm{P}=0.04$ ) and morphine (from $16 \%$ to $8 \% ; \mathrm{P}=0.01$ ) becoming less frequently involved, and methadone (from $10 \%$ to $17 \%$; $\mathrm{P}=0.02$ ) becoming more frequently involved (table 4). Furthermore, $37.8 \%$ (375/993) of people with active opioid prescriptions also had evidence on postmortem toxicology of an opioid that was not prescribed to them. By 2016, the most common non-prescribed opioid involved in these deaths was fentanyl (41\%; 47/115). Finally, among people with no active opioid prescription at time of death, the contribution of various opioids changed significantly over time.
By 2016, nearly half $(47.5 \% ; 274 / 577)$ of these deaths involved fentanyl (compared with $20 \%$ in 2013; $\mathrm{P}<0.001$ ), whereas involvement of oxycodone (from 22\% to 17\%; $\mathrm{P}=0.02$ ), methadone (from $27 \%$ to $18 \%$; $\mathrm{P}<.001$ ), and codeine (from $21 \%$ to $12 \%$; $\mathrm{P}<.001)$ fell over this same period. It is important to note that only a small number $(<3 \%)$ of methadone prescriptions active at time of death were for a pain indication, highlighting the role of methadone used to treat opioid use disorder in these deaths. In an exploratory analysis, among decedents in 2016 in whom fentanyl was detected after death but for whom no active fentanyl prescription was present at time 
of death, just $2 \%(6 / 321)$ were dispensed a fentanyl prescription in the six months preceding death.

In our secondary analysis exploring opioid prescribing before 12847 emergency department visits for an opioid toxicity, our results were generally consistent with those from the mortality analysis. Specifically, we found a significant reduction in previous dispensations of active opioid prescription between 2013 and 2016 (from 44.2\% to 39.5\%; $\mathrm{P}<0.001$; table 5). In 2016, 80.0\% (3058/3821) of people visiting an emergency department for an opioid toxicity event had received a prescription opioid in the previous three years.

\section{Discussion}

In this population based cohort study of nearly 3000 opioid related deaths, we found that in 2016, whereas only one third of patients had an active opioid prescription at time of death, more than three quarters of decedents had been dispensed an opioid in the three years before death. Furthermore, $40 \%$ of the deaths with evidence of an active opioid prescription had additional opioids involved in the death, suggesting an important contribution of either remotely prescribed or illicitly obtained drugs in these deaths. Finally, the role of non-prescribed fentanyl in opioid related deaths increased substantially between 2013 and 2016, among both people actively being prescribed opioids and those with no evidence of prescription opioid use. These findings highlight the important inter-relations between the prescribed and illicit opioid markets among people dying due to opioids in Ontario and the growing role of clandestinely manufactured fentanyl in these trends.

\section{Comparison with other studies}

Our findings are similar to those reported in other jurisdictions, including in British Columbia and Alberta, Canada. In British Columbia, Gladstone et al reported the proportion of deaths related to prescription opioids in 2013 in which an opioid had been prescribed in the previous 60 days. ${ }^{25}$ In this analysis, women were more likely than men to have been recently dispensed an opioid ( $54 \% v 29 \%$ ), which aligns closely with our findings of $48 \%$ of women and $33 \%$ of men having an active opioid prescription at time of death that same year. Similarly, in Alberta, 32\% of opioid related deaths in 2017 occurred in people dispensed an opioid in the previous 30 days, ${ }^{26}$ which is slightly lower than our finding of $43 \%$ in 2016 . This could reflect inter-jurisdictional differences in the role of non-prescribed opioids in fatal overdoses, which has been shown elsewhere, ${ }^{16}$ or might indicate growth in overdoses attributable to clandestinely manufactured synthetic opioids in 2017. ${ }^{27}$ Importantly, in Alberta in 2017 , only $23 \%$ of deaths involving fentanyl had an opioid prescription in the previous 30 days. ${ }^{26}$

An emerging concern across North America is the contamination of the illicit opioid supply with synthetic fentanyl and its analogues. ${ }^{151627}$ Given the potency of these drugs, the lack of regulation and control over the amount consumed, and the proliferation of counterfeit pills containing fentanyl, ${ }^{27}$ jurisdictions across the US and Canada have reported large increases in fentanyl related deaths in 2016 and 2017. ${ }^{15171826}$ In our study, we found evidence of the increasing contributions of fentanyl and its analogues to opioid related deaths in Ontario. In particular, among people who died of an opioid related cause but had no active opioid prescriptions at time of death, only $20 \%$ of deaths involved fentanyl in 2013 compared with nearly 50\% in 2016. Although this clearly shows the growing role of non-prescribed fentanyl in these deaths across the province, it is important to highlight the large contribution of prescribed and diverted prescription opioids to these deaths. Half of the deaths without active opioid prescriptions involved non-fentanyl opioids, suggesting diversion of prescriptions, and approximately $20 \%$ of people who died of an opioid related cause in 2016 had only prescribed opioids in their system at time of death. Finally, the absolute number of people with an active opioid prescription who died of an overdose increased by $15 \%$ over our study period. This reinforces the need for responses to the opioid crisis that address all avenues through which people can access these drugs, including prescriptions, diverted drugs, and illicitly manufactured products.

\section{Strengths and limitations of study}

This study has several strengths, including its use of medical coronial data to identify postmortem toxicological findings for all opioid related deaths occurring in Ontario and the use of comprehensive, linked prescription data to determine the contribution of prescription opioids to these deaths. However, several limitations warrant emphasis. For example, we

\begin{tabular}{|c|c|c|c|c|c|}
\hline Measures & $2013(n=2773)$ & $2014(n=2998)$ & $2015(n=3255)$ & $2016(n=3821)$ & $P$ value (trend test) \\
\hline Active opioid prescription on date of death & $1227(44.2)$ & $1353(45.1)$ & $1440(44.2)$ & $1511(39.5)$ & $<0.001$ \\
\hline Opioid prescription in previous: & & & & & \\
\hline 30 days & $1450(52.3)$ & $1569(52.3)$ & $1718(52.8)$ & $1874(49.0)$ & 0.01 \\
\hline 180 days & $1801(64.9)$ & $1936(64.6)$ & $2161(66.4)$ & $2379(62.3)$ & 0.06 \\
\hline 1 year & $-{ }^{\star}$ & $2148(71.6)$ & $2385(73.3)$ & $2665(69.7)$ & 0.06 \\
\hline 2 years & $-{ }^{*}$ & $-^{\star}$ & $2560(78.6)$ & $2931(76.7)$ & $0.05 t$ \\
\hline 3 years & $-{ }^{*}$ & $-^{*}$ & $-{ }^{*}$ & $3058(80.0)$ & - \\
\hline
\end{tabular}


could not examine more remote opioid prescriptions, and we cannot exclude the possibility that some deaths seeming to involve diverted opioids might have involved older prescriptions to the same person. We characterised this by doing sensitivity analyses, investigating any opioid prescriptions dispensed in the previous three years (when data allowed). Secondly, we cannot differentiate between diverted prescription fentanyl patches and illicitly produced fentanyl by using postmortem toxicology. However, in an exploratory analysis, we confirmed that less than $2 \%$ of people with fentanyl present on toxicology at time of death and no active fentanyl prescription had been dispensed fentanyl at any point in the previous six months. This, in conjunction with the introduction of fentanyl patch-for-patch programmes across Ontario that were designed to reduce diversion of fentanyl patches, ${ }^{28}{ }^{29}$ suggests that the recent growth of involvement of fentanyl in deaths is most likely attributable to illicitly manufactured fentanyl. Finally, we defined heroin involvement in deaths on the basis of presence of 6-monoacetylmorphine in postmortem toxicology. However, owing to its rapid metabolism, this may underestimate the true prevalence of heroin involvement in deaths.

\section{Conclusions and policy implications}

The prescribed and illicit opioid environment continues to evolve, with recent focus on the important role of illicitly produced fentanyl in rising rates of opioid related deaths. This study of all opioid related deaths in Ontario over a four year period describes the ongoing combined roles of prescribed, diverted, and illicit opioids in this market and highlights the emerging contributions of non-prescribed fentanyl to fatal overdoses across the province. Although more than half of all opioid related deaths still involved prescription drugs (either dispensed or diverted) in 2016, the increased prevalence of deaths involving non-prescribed fentanyl between 2015 and 2016 is alarming. In light of these trends, the success of programmes and policies designed to tackle the ongoing opioid crisis cannot focus solely on the prescribed or illicit environment but must consider the multifactorial contributions of various sources of opioids to this public health problem.

We thank the Office of the Chief Coroner for Ontario, which, as part of its public safety mandate, made available the relevant data on opioid related deaths in Ontario. We also thank Brogan, Ottawa, for use of its Drug Product and Therapeutic Class Database.

Contributors: TG, WK, DM, MT, MMM, JMP, and DNJ were involved in the conception and design of the study. TG and WK acquired the data. TG, WK, DM, MT, MMM, JMP, and DNJ analysed and interpreted the data. TG drafted the article. WK, DM, MT, MMM, JMP, and DNJ revised the article critically for important intellectual content. TG, WK, DM, MT, MMM, JMP, and DNJ gave final approval of the version to be published. All authors had access to the data in the study and take responsibility for the integrity of the data and the accuracy of the data analysis. TG attests that all listed authors meet authorship criteria and that no others meeting the criteria have been omitted. She is the guarantor.

Funding: This study was supported by a grant from the Ontario Ministry of Health and Long-Term Care (MOHLTC; grant \#06673) and the Canadian Institutes for Health Research (grant \#153070). It was also supported by the Institute for Clinical Evaluative Sciences (ICES), a non-profit research institute sponsored by the Ontario MOHLTC. The opinions, results, and conclusions reported in this paper are those of the authors and are independent from the funding sources. No endorsement by ICES or the Ontario MOHLTC is intended or should be inferred.

Competing interests: All authors have completed the ICMJE uniform disclosure form at www.icmje.org/coi disclosure.pdf (available on request from the corresponding author) and declare: TG received grant funding from the Ontario Ministry of Health and Long-Term Care to support this work; MMM has received personal fees from Celgene, NovoNordisk, and Allergan outside the submitted work; no other relationships or activities that could appear to have influenced the submitted work.

Ethical approval: The study protocol was approved by the research ethics board of Sunnybrook Health Sciences Centre, Toronto, Ontario. Data sharing: The dataset from this study is held securely in coded form at the Institute for Clinical Evaluative Sciences (ICES). Although data sharing agreements prohibit ICES from making the dataset publicly available, access may be granted to those who meet prespecified criteria for confidential access, available at www.ices.on.ca/ DAS.

Transparency: The lead author (the manuscript's guarantor) affirms that this manuscript is an honest, accurate, and transparent account of the study being reported; that no important aspects of the study have been omitted; and that any discrepancies from the study as planned (and, if relevant, registered) have been explained.

This is an Open Access article distributed in accordance with the Creative Commons Attribution Non Commercial (CC BY-NC 4.0) license, which permits others to distribute, remix, adapt, build upon this work non-commercially, and license their derivative works on different terms, provided the original work is properly cited and the use is noncommercial. See: http://creativecommons.org/licenses/by-nc/4.0/

1 Juurlink DN, Dhalla IA. Dependence and addiction during chronic opioid therapy. J Med Toxicol 2012;8:393-9. doi:10.1007/s13181012-0269-4

2 Gomes T, Mamdani MM, Dhalla IA, Paterson JM, Juurlink DN. Opioid dose and drug-related mortality in patients with nonmalignant pain. Arch Intern Med 2011;171:686-91. doi:10.1001/ archinternmed 2011.117

3 Bohnert AS, Valenstein M, Bair MJ, et al. Association between opioid prescribing patterns and opioid overdose-related deaths. JAMA 2011:305:1315-21. doi:10.1001/jama.2011.370

4 Park TW, Saitz R, Ganoczy D, Ilgen MA, Bohnert AS Benzodiazepine prescribing patterns and deaths from drug overdose among US veterans receiving opioid analgesics: case-cohort study. BM/ 2015:350:h2698. doi:10.1136/bmj.h2698

5 Gomes T, Mastorakos A, Paterson JM, et al, Canadian Network for Observational Drug Effect Studies Investigators. Changes in the dispensing of opioid medications in Canada following the introduction of a tamper-deterrent formulation of long-acting oxycodone: a time series analysis. CMAJ Open 2017;5:E800-7. doi:10.9778/cmajo.20170104

6 Gomes T, Pasricha S, Martins T, et al. Behind the Prescriptions: A snapshot of opioid use across all Ontarians. Ontario Drug Policy Research Network, 2017. doi:10.31027/ODPRN.2017.04.

7 U.S. Centers for Disease Control and Prevention. Opioid overdose. 2017. https://www.cdc.gov/drugoverdose/index.html.

8 Special Advisory Committee on the Epidemic of Opioid Overdoses. National report: Apparent opioid-related deaths in Canada (December 2017). Public Health Agency of Canada, 2017

9 Gomes T, Greaves S, Tadrous M, Mamdani MM, Paterson JM, Juurlink DN. Measuring the Burden of Opioid-related Mortality in Ontario, Canada. J Addict Med 2018. doi:10.1097/ ADM.0000000000000412

10 Dowell D, Haegerich TM, Chou R. CDC Guideline for Prescribing Opioids for Chronic Pain--United States, 2016. JAMA 2016;315:1624-45. doi:10.1001/jama.2016.1464

11 Busse JW, Craigie S, Juurlink DN, et al. Guideline for opioid therapy and chronic noncancer pain. CMAl 2017;189:E659-66. doi:10.1503/cmaj.170363

12 Ontario Ministry of Health and Long-Term Care. Ontario's narcotics strategy: Change in funding status of oxycodone controlled release tablet. 2015. http://www.health.gov.on.ca/en/public/programs/ drugs/ons/oxy_faq.aspx.

13 Baldwin N, Gray R, Goel A, Wood E, Buxton JA, Rieb LM. Fentanyl and heroin contained in seized illicit drugs and overdose related deaths in British Columbia, Canada: An observational analysis. Drug Alcohol Depend 2018;185:322-7. doi:10.1016/j. drugalcdep.2017.12.032 
14 Rubin R. Illicit Fentanyl Driving Opioid Overdose Deaths. IAMA 2017;318:2174.

15 Ciccarone D. Fentanyl in the US heroin supply: A rapidly changing risk environment. Int J Drug Policy 2017;46:107-11. doi:10.1016/j. drugpo.2017.06.010

16 O'Donnell JK, Halpin J, Mattson CL, Goldberger BA, Gladden RM. Deaths Involving Fentanyl, Fentanyl Analogs, and U-47700 - 10 States, July-December 2016. MMWR Morb Mortal Wkly Rep 2017:66:1197-202. doi:10.15585/mmwr.mm6643e1

17 Slavova S, Costich JF, Bunn TL, et al. Heroin and fentanyl overdoses in Kentucky: Epidemiology and surveillance. Int J Drug Policy 2017:46:120-9. doi:10.1016/i.drugpo.2017.05.051

18 British Columbia Coroners Service. Fentanyl-Detected Illicit Drug Overdose Deaths January 1, 2012 to December 31, 2017. Office of the Chief Coroner, 2018.

19 Public Health Ontario. Opioid-related morbidity and mortality in Ontario. 2018. https://www.publichealthontario.ca/en/ dataandanalytics/pages/opioid.aspx.

20 Fischer B, Voitila L, Rehm I. The 'fentanyl epidemic' in Canada Some cautionary observations focusing on opioid-related mortality. Prev Med 2018;107:109-13. doi:10.1016/j.ypmed.2017.11.001

21 Government of Canada. Actions on Opioids: 2016 and 2017. Health Canada, 2017.

22 Cheng T, DeBeck K. Between a rock and a hard place: Prescription opioid restrictions in the time of fentanyl and other street drug adulterants. Can J Public Health 2017;108:e325-7. doi:10.17269/ cjph. 108.6075
23 Barry CL. Fentanyl and the Evolving Opioid Epidemic: What Strategies Should Policy Makers Consider? Psychiatr Serv 2018;69:100-3. doi:10.1176/appi.ps.201700235

24 Dhalla IA, Mamdani MM, Sivilotti ML, Kopp A, Qureshi O, Juurlink DN. Prescribing of opioid analgesics and related mortality before and after the introduction of long-acting oxycodone. CMAl 2009;181:891-6. doi:10.1503/cmaj.090784

25 Gladstone EJ, Smolina K, Weymann D, Rutherford K, Morgan SG. Geographic Variations in Prescription Opioid Dispensations and Deaths Among Women and Men in British Columbia, Canada. Med Care 2015;53:954-9. doi:10.1097/MLR.0000000000000431

26 Alberta Health. Opioids and Substances of Misuse: Alberta Report, 2017 Q3. Government of Alberta, 2017.

27 Fairbairn N, Coffin PO, Walley AY. Naloxone for heroin, prescription opioid, and illicitly made fentanyl overdoses: Challenges and innovations responding to a dynamic epidemic. Int J Drug Policy 2017;46:172-9. doi:10.1016/j.drugpo.2017.06.005

28 Government of Ontario. Safeguarding our Communities Act (Patch for Patch Return Policy), 2015, S.0. 2015, c. 33, 2016.

29 Ontario College of Pharmacists. Patch-For-Patch Fentanyl Return Program: Fact Sheet. 2016. http://www.ocpinfo.com/regulationsstandards/policies-guidelines/Patch_For_Patch_Fentanyl_Return Fact_Sheet/.

\section{Supplementary materials}

